\title{
A Wafer-Bonded, Floating Element Shear-Stress Sensor Using a Geometric Moiré Optical Transduction Technique
}

\section{Stephen Horowitz, Tai-An Chen, Venkataraman Chandrasekaran*, Ken Tedjojuwono ${ }^{\S}$, Louis Cattafesta, Toshikazu Nishida, and Mark Sheplak ${ }^{\dagger}$}

\author{
Interdisciplinary Microsystems Group, PO Box 116250, University of Florida, Gainesville, Florida 32611-6250 \\ † (352) 392-3983, FAX: (352) 392-7303, ms@mae.ufl.edu \\ *Department of Aeronautics and Astronautics, MIT,
Cambridge, MA 02139

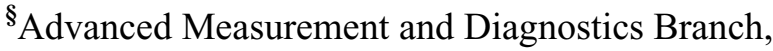 NASA-LaRC, Hampton, VA 23681-2199}

\section{ABSTRACT}

This paper presents a geometric Moiré optical-based floating-element shear stress sensor for wind tunnel turbulence measurements. The sensor was fabricated using an aligned waferbond/thin-back process producing optical gratings on the backside of a floating element and on the top surface of the support wafer. Measured results indicate a static sensitivity of $0.26 \mu \mathrm{m} / \mathrm{Pa}$, a resonant frequency of $1.7 \mathrm{kHz}$, and a noise floor of $6.2 \mathrm{mPa} / \sqrt{\mathrm{Hz}}$.

\section{INTRODUCTION}

The MEMS community has developed both thermal [1] and floating element [2-7] shear-stress sensors. Thermal sensors are robust and simpler to fabricate; however, they are based on a heat transfer analogy and absolute calibration for quantitative measurements is difficult [8]. Conversely, floating-element structures respond directly to wall shear stress and provide the best opportunity to obtain quantitative, time-resolved measurements in a controlled wind tunnel environment. Several transduction methods exist for measurement of the shear-stress induced deflection of the floating element, including capacitive, $[2,3,7]$ piezoresistive, $[4,5]$ and differential optical shutter techniques [6]. An ideal sensor is truly flush-mounted with no wire bonds that generate flow disturbances and is immune to non-shear stress inputs (i.e., electromagnetic interference (EMI), pressure fluctuations, vibrations, etc.). Only one existing device does not possess front-side wire-bonds, but it was not designed for turbulence applications and possesses too low a sensitivity [5]. The optical shutter technique [6] provided immunity to EMI and pressure fluctuations, but it possessed wire bonds and was sensitive to tunnel vibration due to the separation of the light source from the sensor. Additional information comparing these devices is given by Naughton and Sheplak [8] in their review of MEMSbased shear stress sensors.

An alternative transduction technique for measurement of small displacements involves the use of Moiré patterns [9-11]. This technique was specifically applied for motion detection of MEMS structures by Tran et al. [11], who reported a $50 \mathrm{~nm}$ displacement resolution using a simple image processing technique with a phase detection resolution of $5^{\circ}$. Our device employs an optical geometric Moiré transduction technique for measurement of shear-stress induced floating-element displacement. The geometric Moiré floating element sensor (Figure 1) possesses immunity from EMI and transverse element movement due to pressure fluctuations and/or vibrations. The flow disturbance is minimal because the incident and reflected light comes through the backside of the Pyrex wafer.

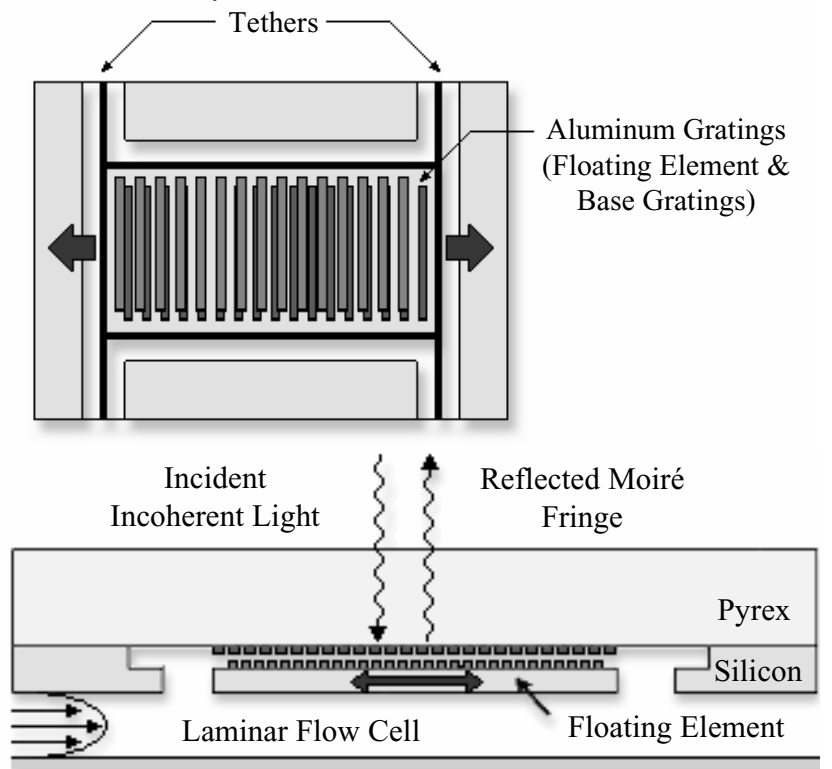

Figure 1: Top view and cross sectional schematic of the firstgeneration optical shear-stress sensor. Note: drawings not to scale.

\section{SENSOR DESIGN}

A schematic of a generic MEMS floating element structure is given in Figure 2. The floating element possesses a length, $L_{e}$, width, $W_{e}$, and thickness, $t$. The floating element is suspended over a recessed gap $g$ by silicon tethers that also serve as restoring springs. The displacement $\delta$ of the floating element as a function of wall shear stress, $\tau_{w}$, is determined via EulerBernoulli beam theory to be

$$
\delta=\tau_{w} \frac{L_{e} W_{e}}{4 E t}\left(\frac{L_{t}}{W_{t}}\right)^{3}\left\{1+2 \frac{L_{t} W_{t}}{L_{e} W_{e}}\right\},
$$

where $L_{t}$ is the tether length, $W_{t}$ is the tether width, and $E$ is the elastic modulus of the tether [2]. Euler-Bernoulli beam theory assumes small deflections such that the strain at the neutral axis of

Travel support has been generously provided by the Transducers Research Foundation and by the DARPA MEMS and DARPA BioFlips programs. 
the beam can be neglected. The limits of this approximation can be approximated via a large-deflection energy-based solution,

$$
\delta\left[1+\left(\frac{3}{4}\right)\left(\frac{\delta}{W_{t}}\right)^{2}\right]=\frac{\tau_{w} W_{e} L_{e}}{4 E t}\left[\frac{L_{t}}{W_{t}}\right]^{3}\left[1+2 \frac{W_{t} L_{t}}{W_{e} L_{e}}\right] .
$$

It is clear that if the maximum deflection of the floating element is a significantly less than the tether width, then Eq. (2) reduces to the small deflection solution given in Eq. (1).
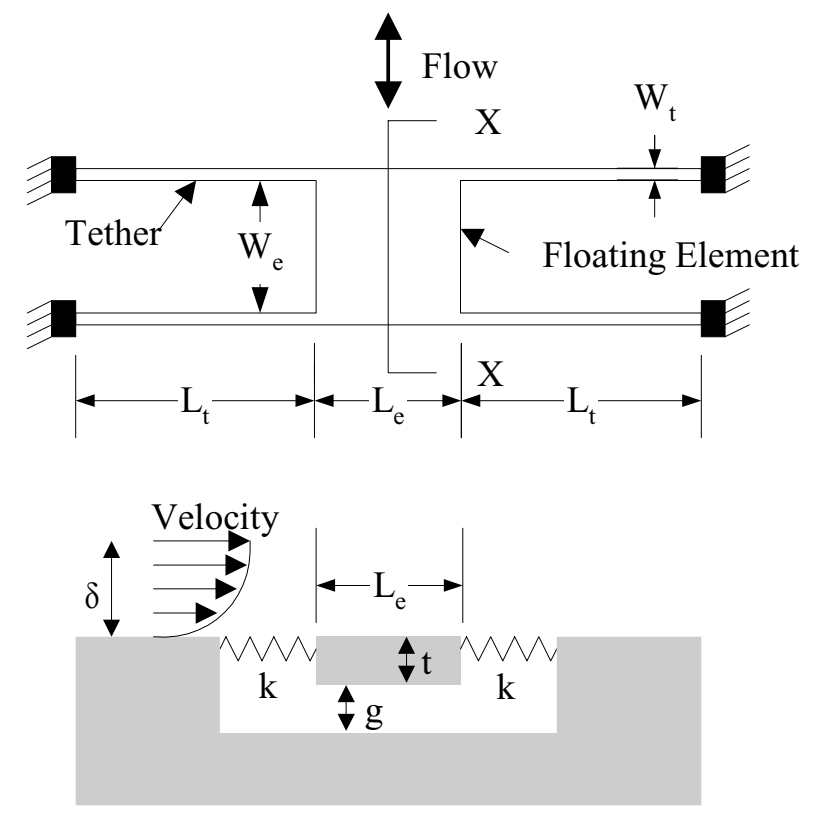

Figure 2: Schematic plan view and cross-section of a typical floating-element sensor.

The element possesses an effective mass, $M$, and the tethers possess an effective spring constant, $k$. The mechanical sensitivity of the sensor with respect to the integrated shear stress force, $F_{\tau}=\tau_{w} W_{e} L_{e}$, is directly proportional to the compliance of the tethers, $1 / k$, given by

$$
\frac{1}{k}=\frac{\delta}{F_{\tau}}=\frac{1}{4 E t}\left(\frac{L_{t}}{W_{t}}\right)^{3}\left\{1+2 \frac{L_{t} W_{t}}{L_{e} W_{e}}\right\} .
$$

The compliance of the device will be limited either by failure at the maximum applied shear stress or from the onset of geometric nonlinearities in the force-displacement relationship shown in Eq. (2). The requirement of high spatial resolution requires the measurement of very small forces. For example, a sensor possessing a $100 \mu^{2}$ floating element structure would need to measure a $10 \mathrm{pN}$ force to resolve a shear stress level of $1 \mathrm{mPa}$, thus requiring a highly compliant structure. If $L_{e} W_{e}>L_{t} W_{t}$, then the effective mass is approximated by $M \approx \rho L e^{W_{e} t}$, where $\rho$ is the mass-averaged density of the element material. Assuming a perfectly-damped or under-damped system, the bandwidth is proportional to the first resonance of the device, $\sqrt{k / M}$. Therefore, the shear-stress sensitivity-bandwidth product for the device is proportional to

$$
\frac{1}{\sqrt{k M}} \propto \sqrt{\frac{1}{4 E \rho L_{e} W_{e} t^{2}}\left(\frac{L_{t}}{W_{t}}\right)^{3}} .
$$

The sensitivity-bandwidth product is a useful figure of merit to investigate the scaling of mechanical sensors analogous to the gain-bandwidth product of an operational amplifier. The increase in the sensitivity-bandwidth figure of merit with decrease in $M$ while maintaining the tether compliance illustrates that MEMSenabled scaling is favorable for the development of low mass, compliant mechanical sensors possessing superior sensitivitybandwidth products relative to conventional sensors. As is the case in all transducers, the minimum detectable signal will be determined by the electronic and/or thermal-mechanical noise floor of the measurement system [12]. The favorable miniaturization scaling of the mechanics of the structure is somewhat mitigated by the requirement to measure very small displacements that can be $\mathrm{O}(\AA)[6]$.

Our device structure consists of a $1280 \mu \mathrm{m} \times 400 \mu \mathrm{m}$ silicon floating element of $10 \mu \mathrm{m}$ thickness, suspended $2.0 \mu \mathrm{m}$ above the surface of a $500 \mu \mathrm{m}$ thick Pyrex wafer by four $545 \mu \mathrm{m} \times 6 \mu \mathrm{m}$ tethers of $10 \mu \mathrm{m}$ thickness. The Moiré pattern is realized by patterning aluminum lines of pitch $g_{2}$ on the bottom of the floating element and $g_{1}$ on the Pyrex wafer. The superimposed top and bottom gratings create a translation-dependent Moiré fringe pattern with spatial period $G$ (Figure 3). The period of the Moiré fringe can be derived by considering that for every Moiré period, the smaller grating has one extra line than the larger grating, thus

$$
G=n \cdot g_{2}=(n+1) g_{1},
$$

where $n$ is the number of lines of the larger grating [9]. By eliminating $n$ from Eq. (5), the spatial period of the Moiré fringe can be given by

$$
G=g_{1} g_{2} /\left(g_{2}-g_{1}\right)
$$

Furthermore, the displacement of the Moiré fringe, $\Delta$, is amplified as compared to the grating displacement $\delta$, as given by

$$
\Delta=\delta\left(\frac{G}{g_{1}}\right) .
$$

Therefore, the Moiré amplification of the sensor can be adjusted by appropriate choice of the grating dimensions. For the design presented here, $G / g_{1}=100$.

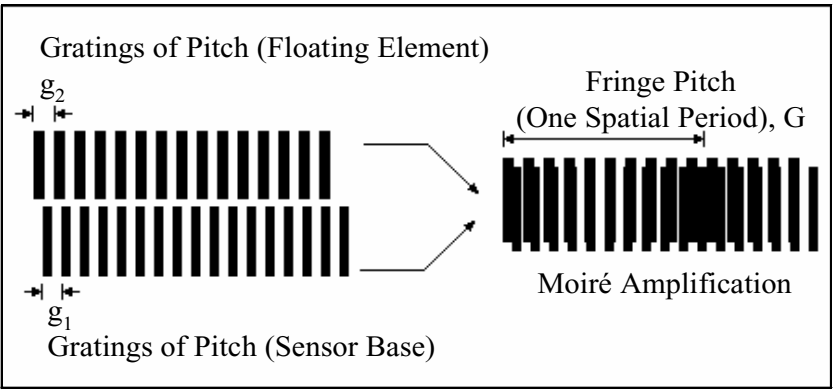

Figure 3: Geometric Moiré pattern generated from two gratings where $g_{1}=9.9 \mu \mathrm{m}, g_{2}=10 \mu \mathrm{m}$, and $G=990 \mu \mathrm{m}$.

\section{SENSOR FABRICATION}

The sensor was fabricated using an aligned wafer-bond/thinback process that produces optical gratings on the backside of a floating element and on the top surface of the support wafer. The wafer-bond/thin-back process is outlined in Figure 4. The process begins with one silicon-on-insulator (SOI) wafer and one Pyrex wafer. First, a $2 \mu \mathrm{m}$ recess is etched into the top silicon layer of 
the SOI wafer using reactive ion etching. This recess will later function as a cavity above which the floating element will move. Aluminum gratings of $0.25 \mu \mathrm{m}$ thickness are then sputter deposited and patterned within the cavity as well as on the top surface of the Pyrex wafer. The inverted SOI wafer is then aligned and anodically bonded to the Pyrex wafer using an EV-501 Wafer Bonder. Following this, the backside of the SOI wafer is thinnedback via potassium hydroxide etching, stopping on the buried oxide (BOX) layer. The BOX layer is then removed prior to deep reactive ion etching that forms the tethers and releases the floating element (Figure 5).

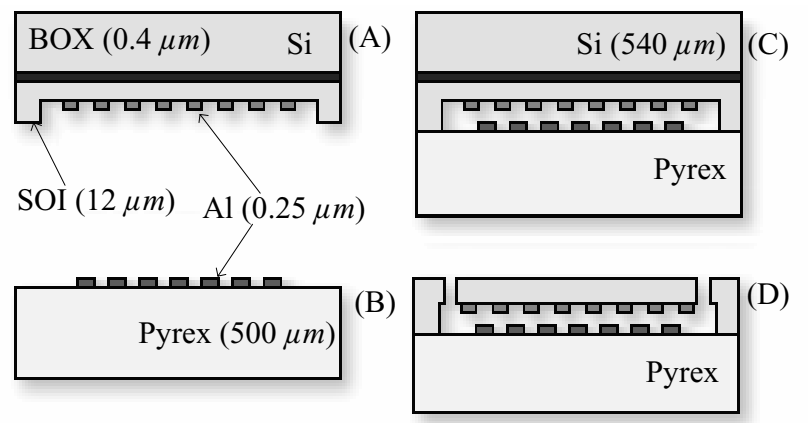

Figure 4: A schematic of the fabrication sequence: (A) Etch $2 \mu \mathrm{m}$ recess in Si-overlayer of SOI wafers, then deposit and pattern device gratings. (B) Deposit and pattern handle gratings on the Pyrex wafer. (C) Align, then anodic bond the Pyrex and SOI wafers. (D) Thin-back the bulk of the SOI wafer, then use DRIE to release the floating element and tethers. Note: schematics not to scale.

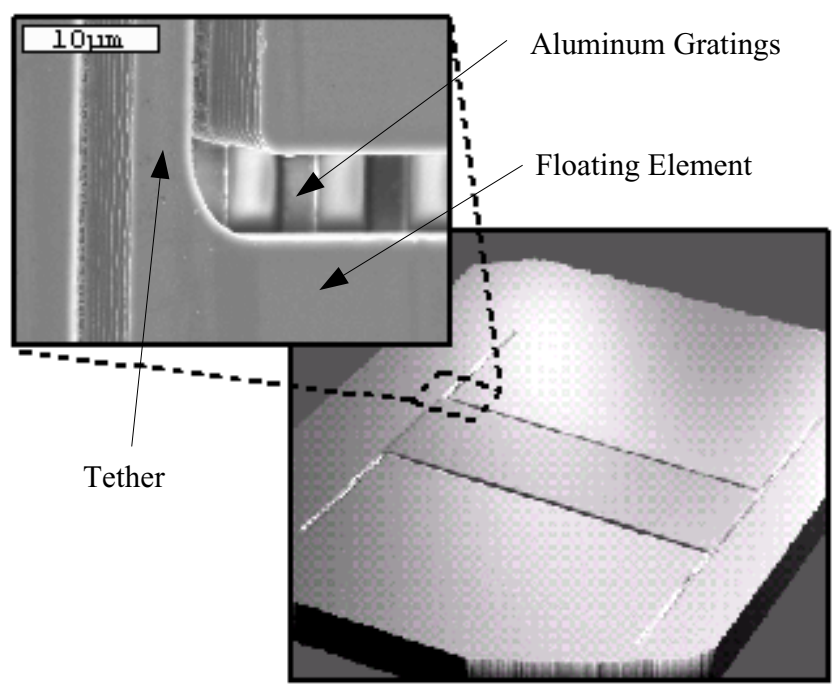

Figure 5: Top-view of the shear stress sensor using an optical profilometer. Inset: Close-up of tether and aluminum gratings taken using scanning-electron microscope (SEM).

The finished device was then packaged by flush-mounting the sensor die in a Lucite plug with back-side imaging optics, providing a $5 \mathrm{x}$ optical magnification, and a Thomson-CSF TH78CE13 linescan CCD camera (Figure 6). The CCD camera contains an array of $1 \times 1024$ pixels, each $10 \mu \mathrm{m}$ in width.

\section{RESULTS AND DISCUSSION}

For static calibration, the packaged device was then mounted into a $100 \mathrm{~mm} \times 1 \mathrm{~mm}$ flow cell that provides a variable mean shear stress via a laminar, incompressible, fully developed, 2-D pressure driven flow in a slot [13] (Figure 6). A block diagram of the static calibration procedure is given in Figure 7 . The differential pressure between two locations in the fully-developed region of the laminar flow cell is measured via a Heise pressure sensor, which is then used to compute the applied shear stress to the sensor. This differential pressure measurement is averaged 100 times and used to compute the applied shear stress $\tau_{w}$ via

$$
\tau_{w}=-\frac{h}{2} \frac{\Delta p}{L},
$$

where $\Delta p$ is the differential pressure, $h$ is the height of the channel, and $L$ is the distance separating the pressure ports.

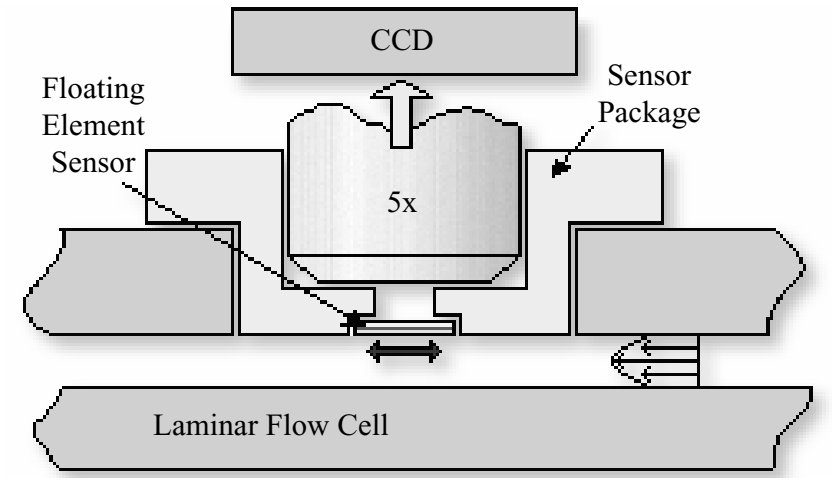

Figure 6: Schematic diagram of static calibration experimental setup illustrating backside imaging optics for 2-D laminar flow cell.

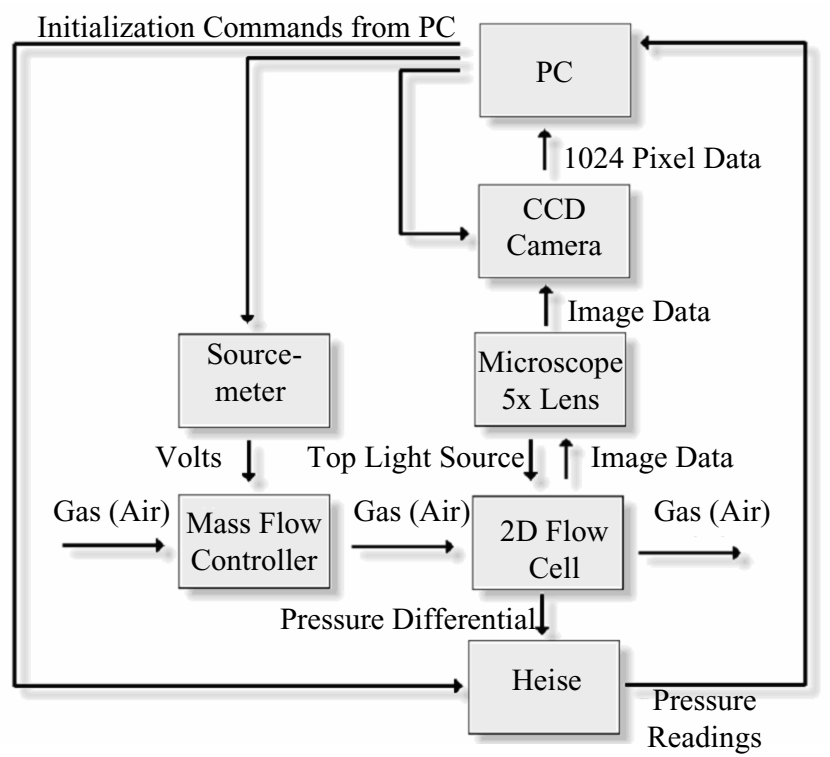

Figure 7: Schematic diagram of experimental setup for static calibration.

The Moiré fringe pattern is captured using the CCD camera, through the backside imaging optics. For the static calibration, 600 successive frames are obtained for averaging purposes. The imaged fringe pattern for zero applied shear stress is shown in Figure 8 , where the first 100 successive frames from the camera are stacked vertically. The peak in relative intensity corresponds to the brightest region in the grayscale image.

From the captured image, relative pixel intensities are obtained for each frame and averaged over the 600 frames. The 
averaged relative pixel intensities obtained for two different shear stresses are shown in Figure 9. The Moiré pattern was found to have a spatial period of $1002 \mu \mathrm{m}$, before the $5 \mathrm{x}$ optical amplification, compared to the physical grating period of $9.9 \mu \mathrm{m}$. The Moiré amplification for the sensor was then found to be 101.2 compared to our designed value of $G / g_{1}=100$.

The resulting averaged intensity pattern is normalized by a calibration image to eliminate pixel gain variations and nonuniform illumination effects. Following this, a spatial Fast-Fourier Transform (FFT) is performed on the normalized fringe pattern, from which the phase of the Moire pattern is then extracted. This phase is then compared against the phase calculated for zero applied shear stress to obtain the phase shift and the corresponding pixel shift. Using knowledge of the Moiré pattern and optical magnification, the corresponding mechanical displacement of the floating element is computed to give a direct measurement of the wall shear stress.

Following the procedure outlined above, the pixel shift of the Moiré fringe pattern was determined for a range of applied shear-stress. The results are shown in Figure 10, along with the corresponding mechanical displacement. The mechanical sensitivity, as found from the slope of this curve, is $0.26 \mu \mathrm{m} / \mathrm{Pa}$, while the Moire fringe, after the $5 \mathrm{x}$ optical amplification, moves by $130.02 \mu \mathrm{m} / \mathrm{Pa}$. Figure 10 illustrates a linear response up to $1.3 \mathrm{~Pa}$.

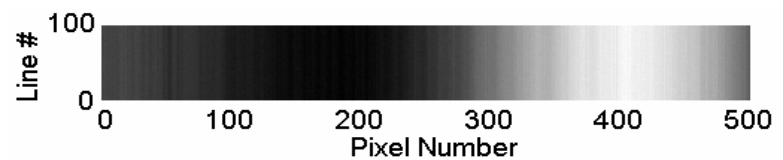

Figure 8: Moiré fringe pattern for a static shear stress of $0 \mathrm{~Pa}$ as seen by 1024 pixel linescan camera. Successive frames from the camera are stacked vertically.

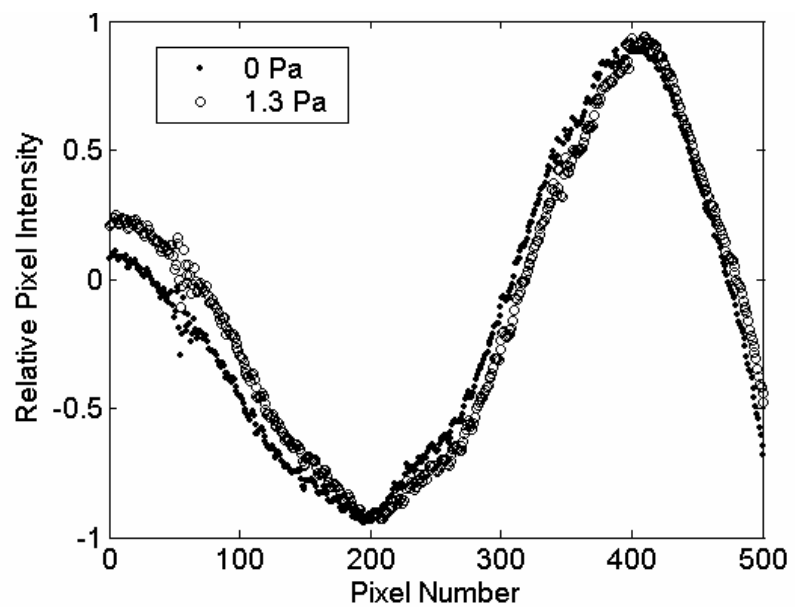

Figure 9: Measured relative pixel intensity for mean shear stresses of $0 \mathrm{~Pa}$ and $1.3 \mathrm{~Pa}$.

The recessed gap under the floating element gives rise to pressure-gradient induced errors. The magnitude of the effective shear-stress, $\tau_{\text {eff }}$, acting in the presence of a pressure gradient has been shown to be

$$
\tau_{\text {eff }}=\left(1+\frac{g}{h}+\frac{2 t}{h}\right) \tau_{w},
$$

where $h$ is the channel height of the wind tunnel used for calibration, $g$ is the recessed gap, and $\tau_{w}$ is the actual wall shear stress [2]. The second and third terms in the bracket are the error terms associated with flow under the floating element and the pressure gradient acting on the lip of the element, respectively. For the current device and experimental apparatus, this component of the calibration error is $2 \%$.

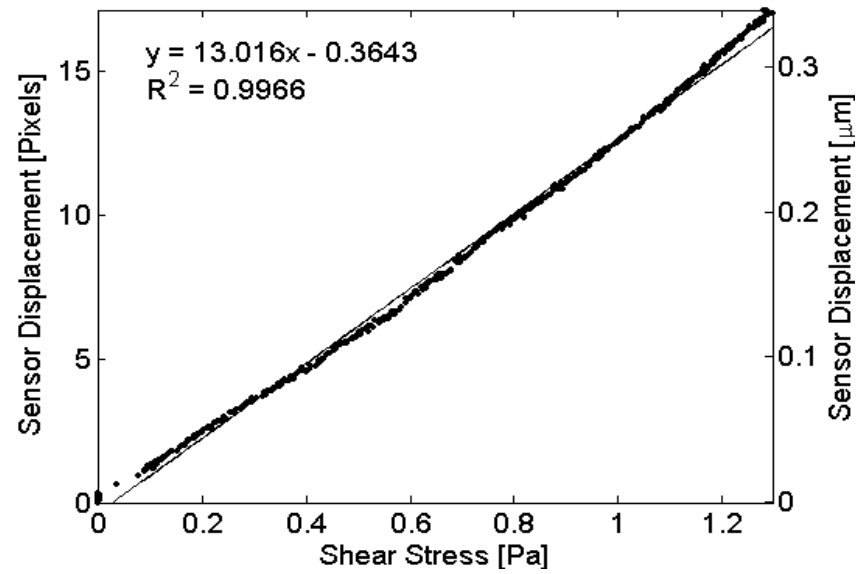

Figure 10: Static response of the sensor in terms of Moiré fringe pixel displacement and corresponding mechanical displacement as a function of mean shear stress. The static sensitivities are 13.0 pixels/Pa and $0.26 \mu \mathrm{m} / \mathrm{Pa}$.

Dynamic calibration was performed in a plane-wave tube, with a $25.4 \mathrm{~mm} \times 25.4 \mathrm{~mm}$ cross section, using a Stokes Layer excitation technique (Figure 11). This technique utilizes acoustic plane waves in a duct to generate known oscillating wall shear stresses [14]. The plane waves are generated by a JBL $2426 \mathrm{H}$ speaker mounted to the end of the plane wave tube. The packaged shear stress sensor is flush mounted to the side wall of the plane, wave tube directly across from a Brüel \& Kjær 4138 1/8" microphone. As with the static setup, the Moiré pattern is recorded by the CCD camera. In the dynamic calibration, however, the camera is programmed to record 16,384 lines at a line rate of 11.42 $\mathrm{kHz}$. The microphone signal is recorded by a data acquisition (DAQ) card via an SRS-560 preamplifier for AC coupling. A synchronization pulse from the camera is used to trigger sampling by the DAQ card.

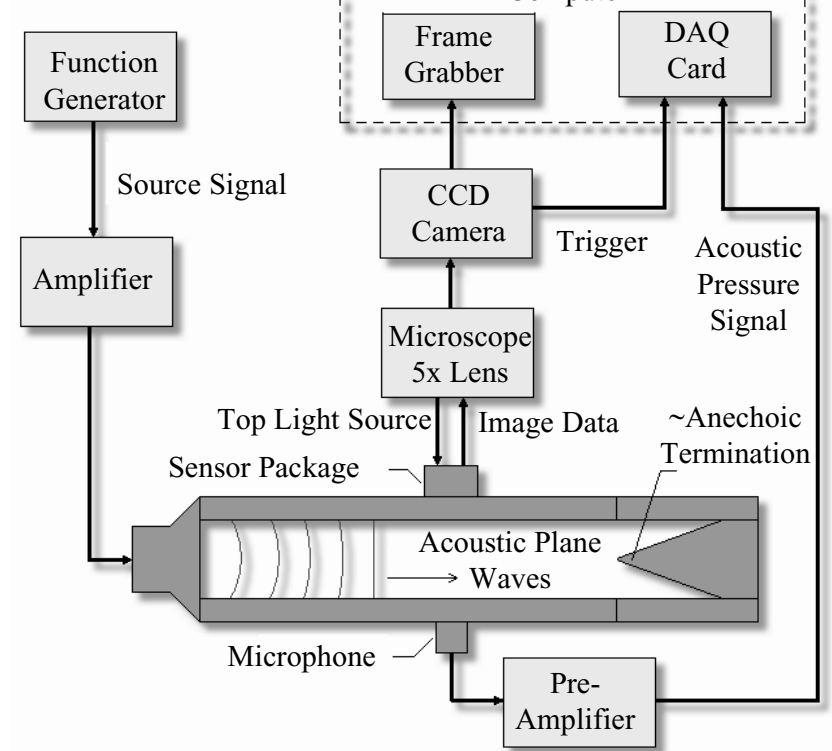

Figure 11: Schematic diagram of experimental setup for dynamic calibration via Stokes' Layer Excitation [14]. 
The recorded Moiré fringe pattern image for a sinusoidal shear stress of amplitude $0.061 \mathrm{~Pa}$ and frequency of $1 \mathrm{kHz}$ is shown in Figure 12. This image shows 40 successive lines from the line scan camera stacked vertically, illustrating temporal oscillations of the phase of the fringe pattern. The sinusoidal time trace on the right represents the input oscillatory shear stress.

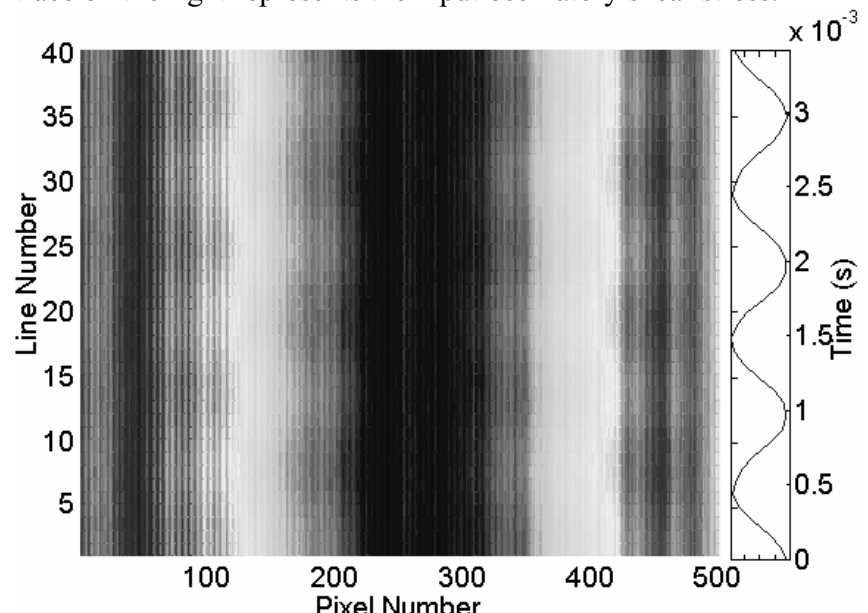

Figure 12: Moiré fringe pattern for a $1 \mathrm{kHz}$ sinusoidal shearstress input of $0.061 \mathrm{~Pa}$. Successive frames from the camera are stacked vertically illustrating the oscillatory fringe pattern. The sinusoidal time trace on the right represents input oscillatory shear stress.

Time series data illustrating the dynamic response of the sensor in terms of Moiré fringe pixel displacement and corresponding mechanical displacement for a $1 \mathrm{kHz}$ sinusoidal input of $0.061 \mathrm{~Pa}$ is shown in Figure 13. The amplitude of the sensor displacement is approximately 3 pixels or $0.06 \mu \mathrm{m}$.

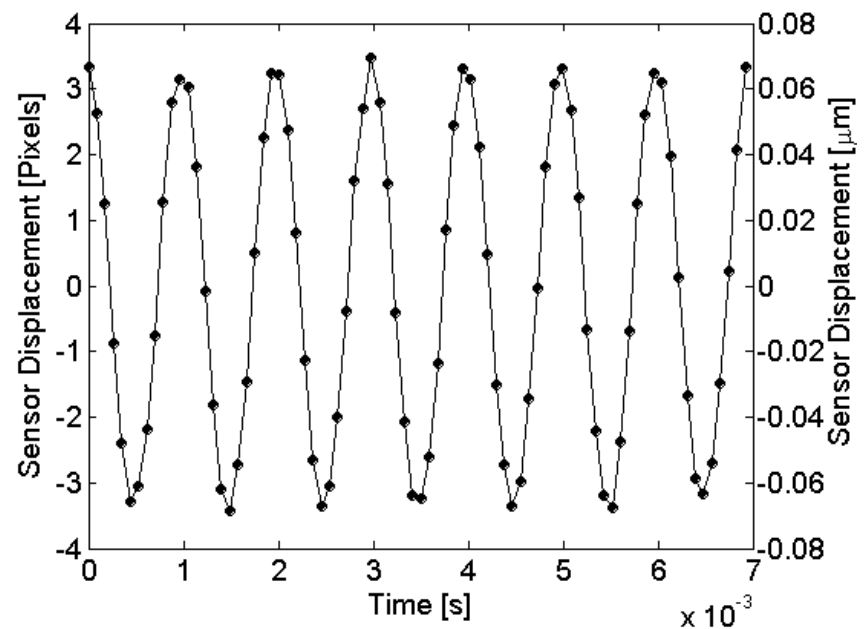

Figure 13: Time series data illustrating the dynamic response of the sensor in terms of Moiré fringe pixel displacement and corresponding mechanical displacement for a $1 \mathrm{kHz}$ sinusoidal input of $0.061 \mathrm{~Pa}$. The dots are the actual samples taken and the line is a curve fit to the data.

From the time series data, the frequency response function can be easily found. A preliminary estimate of the frequency response function gain factor is shown in Figure 14. Results indicate a first lateral mode resonant frequency at $1.7 \mathrm{kHz}$. The corresponding phase has not been accurately estimated due to synchronization issues between the linescan CCD and the microphone channel analog-to-digital converter. This issue arose despite the trigger signal from the CCD camera that is used to trigger the start of microphone sampling. It is believed that roundoff error in the sampling rate of the DAQ card leads to a gradual loss of synchronization over time. Further work is necessary to elucidate the precise nature of this issue.

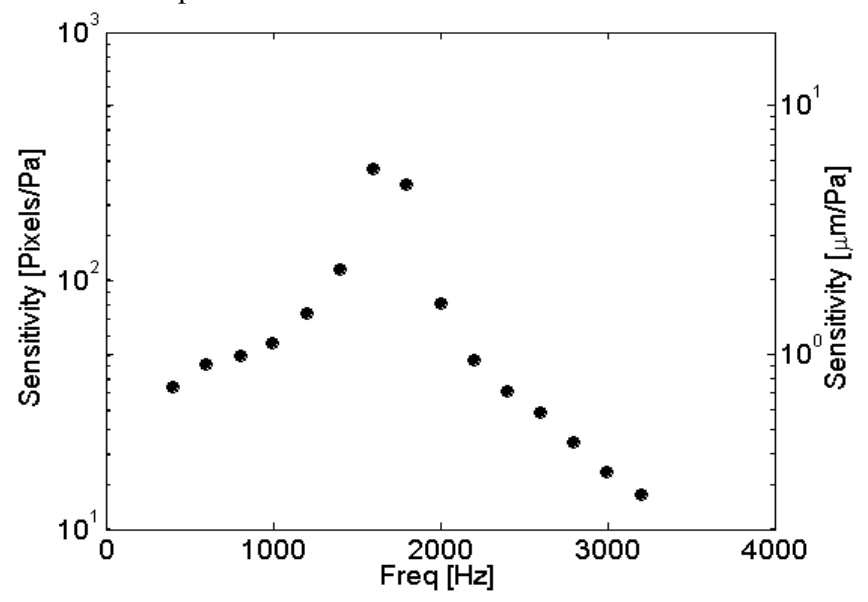

Figure 14: Magnitude of frequency response function illustrating a resonance at approximately $1.7 \mathrm{kHz}$.

The noise floor spectrum was obtained by recording microphone and CCD data with no acoustic input signal. An estimate of the noise floor magnitude is shown in Figure 15. For a $1 \mathrm{~Hz}$ bin centered at $1 \mathrm{kHz}$, the noise floor is $1.6 \mathrm{~nm} / \sqrt{ } \mathrm{Hz}$ or 0.08 pixels $/ \sqrt{ } \mathrm{Hz}$, which corresponds to a minimum detectable shear stress of $6.2 \mathrm{mPa} / \sqrt{ } \mathrm{Hz}$. The dynamic range spans three orders of magnitude $(6.2 \mathrm{mPa}-1.3 \mathrm{~Pa})$.

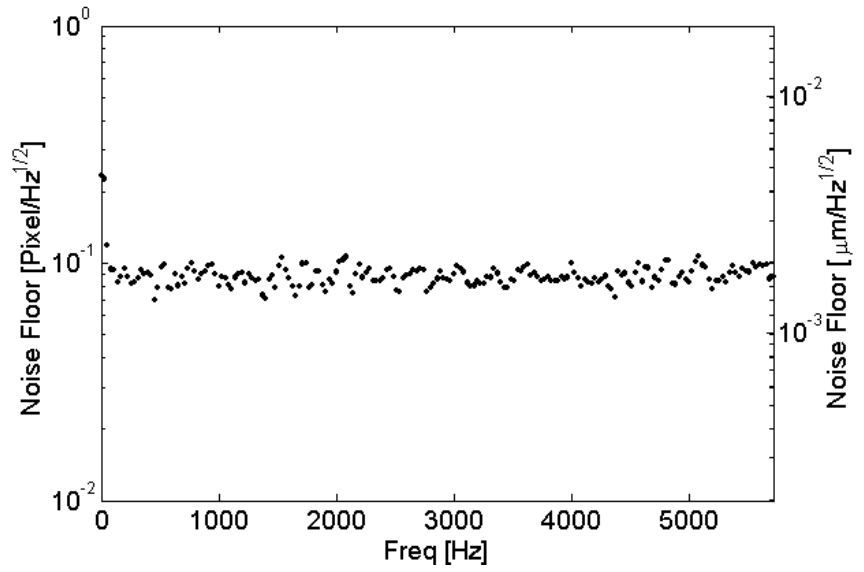

Figure 15: Noise floor spectrum of the sensor with a $1 \mathrm{~Hz}$ binwidth.

\section{CONCLUSIONS AND FUTURE WORK}

A proof-of-concept micromachined, floating element shearstress sensor was developed that utilizes a geometric Moiré interferometric technique. A static characterization of the device indicated a linear response up to $1.3 \mathrm{~Pa}$. Noise floor measurements indicate a minimum detectable shear stress of 6.2 $\mathrm{mPa}$ for a $1 \mathrm{~Hz}$ binwidth. Therefore, the experimentally determined dynamic range is $6.2 \mathrm{mPa}-1.3 \mathrm{~Pa}$. The upper end of the dynamic range as predicted by Eq. (9) for a $3 \%$ static nonlinearity is $21.7 \mathrm{~Pa}$. This, however, could not be verified due to constraints in our static calibration apparatus. A comparison of the specifications of various shear stress sensors including the sensor presented here is given in Table 1.

In the dynamic experiments, the device exhibited a lower resonant frequency than was expected. Fabrication-induced 
geometric errors have not yet been quantified, but this may be the underlying cause for the discrepancy. The measured displacement resolution was found to be $1.6 \mathrm{~nm} / \sqrt{\mathrm{Hz}}$ for the current sensor and optical setup. There are several mechanisms by which this performance can be improved. The sensor and testbed and can be subdivided into three gain stages, consisting of mechanical sensitivity, Moiré amplification, and optical magnification. The mechanical sensitivity of the sensor can be increased but at the expense of decreased resonant frequency, in a typical gain bandwidth tradeoff. Additionally, the Moiré amplification can be increased, through two mechanisms. As the amplification is dependent upon the ratio of $G / g_{1}$, increasing $G$ or decreasing $g_{1}$ has the same overall effect, an increase in Moiré amplification. Nevertheless, it is necessary to have at least one full Moiré period within the field of view of the CCD camera to allow accurate determination of the phase of the Moiré pattern. This places an upper limit upon $G$, and makes the reduction of $g_{1}$ the most desirable method of increasing the Moire amplification. Ultimately, $g_{1}$ and $g_{2}$ are also limited, in this case by the photolithographic resolution of the gratings. Furthermore, as the grating pitch decreases, diffraction effects become more significant, leading to a blurring of the image and a reduction in fringe contrast.

Table 1: Comparison of specifications for various MEMS shearstress sensors. The bandwidth, BW, of each of the sensors is listed in the far right column.

\begin{tabular}{|l|c|c|c|c|}
\hline \multicolumn{1}{|c|}{ Author } & Type & Dyn. Range & Sensitivity & BW \\
\hline Schmidt et al.[2] & Direct & $0.01-1 \mathrm{~Pa}$ & $52 \mathrm{mV} / \mathrm{Pa}$ & $10 \mathrm{kHz}$ \\
\hline Pan et al. [3, 15] & Direct & $0.5-3.8 \mathrm{~Pa}$ & $1 \mathrm{~V} / \mathrm{Pa}$ & $\mathrm{n} / \mathrm{a}$ \\
\hline $\begin{array}{l}\text { Padmanabhan et } \\
\text { al. [6] }\end{array}$ & Direct & $1.4 \mathrm{mPa}-10 \mathrm{~Pa}$ & $0.4 \mathrm{mV} / \mathrm{Pa}$ & $10 \mathrm{kHz}$ \\
\hline Liu et al. [1] & Thermal & $\mathrm{n} / \mathrm{a}$ & $1 \mathrm{v} / \mathrm{Pa}$ & $25 \mathrm{kHz}$ \\
\hline Liu et al. [1] & Thermal & $\mathrm{n} / \mathrm{a}$ & $30 \mathrm{mv} / \mathrm{Pa}$ & $18 \mathrm{kHz}$ \\
\hline Sheplak et al. [14] & Thermal & $9 \mu \mathrm{Pa}-1.7 \mathrm{~Pa}$ & $11 \mathrm{mV} / \mathrm{Pa}$ & $8 \mathrm{kHz}$ \\
\hline Horowitz et al. & Direct & $6.2 \mathrm{mPa}-1.3 \mathrm{~Pa}$ & 13 pixels $/ \mathrm{Pa}$ & $1.7 \mathrm{kHz}$ \\
\hline
\end{tabular}

The final mechanism by which the sensitivity can be increased is via a higher optical magnification. The advantage of this technique is that the sensitivity can be increased even after device processing is completed. The main disadvantage is a corresponding amplification of relative package and optical testbed vibrations, although this can be compensated by improvements in the packaging, and a reduction in optical depth of field. It should be noted that the package vibrations are not amplified by the Moiré amplification as only relative vibrations between the two gratings are amplified. Additionally, the field of view of the camera limits the maximum magnification, as once again it is necessary to have a minimum of one full Moiré period in view.

There are several issues regarding the reliability of the sensor. First, the floating element is designed with a hard stop after $7 \mu \mathrm{m}$ to prevent damage to the sensor due to excessive shear stress. Additionally, temperature issues can play a role in both sensitivity and reliability, however, silicon and Pyrex have similar thermal expansion coefficients, so negligible thermal-induced stresses are expected.

Future work will include more rigorous dynamic and static characterization, noise floor studies, and sensitivity to non-shear stress inputs, as well as resolving the dynamic calibration synchronization issue. In addition, a borescope based imaging system will be implemented to reduce package size and improve portability. A second-generation device is planned in which the sensor geometry and Moiré fringe design will be optimized.

\section{ACKNOWLEDGEMENTS}

The authors would like to thank Professor Peter Ifju, Hamed Kourouma, Jhoanna Peña and Julio Castro for their assistance in this project. This work was supported through NASA-LaRC grant NAG-1-2133 and NASA-KSC grant NAG-10-316.

\section{REFERENCES}

1. C. Liu, J.-B. Huang, Z. Zhu, F. Jiang, S. Tung, Y.-C. Tai, and C.-M. Ho, "A Micromachined Flow Shear-Stress Sensor Based on Thermal Transfer Principles", J.MEMS, 8, (1999), pp. 90-9.

2. M. A. Schmidt, R. T. Howe, S. D. Senturia, and J. H. Haritonidis, "Design and Calibration of a Microfabricated Floating-Element Shear-Stress Sensor", IEEE Tr. on Elect. Dev., 35, 6 (1988), pp. 750-57.

3. T. Pan, D. Hyman, M. Mehregany, E. Reshotko, and S. Garverick, "Microfabricated Shear Stress Sensors, Part 1: Design and Fabrication", AIAA J., 37, (1999), pp. 66-72.

4. J. Shajii, K.-Y. Ng, and M. A. Schmidt, "A Microfabricated Floating-Element Shear Stress Sensor Using Wafer-Bonding Technology", J. MEMS, 1, 2 (1992), pp. 89-94.

5. H. D. Goldberg, K. S. Breuer, and M. A. Schmidt. "A Silicon Wafer-Bonding Technology for Microfabricated Shear-Stress Sensors with Backside Contacts", in Solid-State Sensor and Actuator Workshop, Hilton Head, (1994), pp. 111-5.

6. A. Padmanabhan, H. D. Goldberg, M. A. Schmidt, and K. S. Breuer, "A Wafer-Bonded Floating-Element Shear-Stress Microsensor with Optical Position Sensing by Photodiodes", $J$. MEMS, 5, (1996), pp. 307-15.

7. J. Zhe, K. R. Farmer, and V. Modi. "A MEMS Device for Measurement of Skin Friction Using Capacitance Sensing", in Proc. of IEEE MEMS 01, Berkeley, CA,, (2001), pp. 4-7.

8. J. Naughton and M. Sheplak, "Modern Developments in ShearStress Measurement", Prog. Aero. Sci., 38, (2002), pp. 515-70.

9. D. Post, B. Han, and P. Ifju, High Sensitivity Moiré, NY, Springer (1994).

10. O. Kafri and I. Glatt, The Physics of Moiré Metrology, NY, Wiley (1990).

11. A. Tran, J. Lee, K. Zhang, and Y. Lo, "Ultrafine Motion Detection of Micromechanical Structures Using Optical Moiré Patterns", IEEE Phot. Tech. Letters, 8, 8 (1996), pp. 1058-60.

12. T. Gabrielson, "Mechanical-Thermal Noise in Micromachined Acoustic and Vibration Sensors", IEEE Elect. Dev., 40, (1993), pp. 903-9.

13. M. Sheplak, V. Chandrasekaran, A. Cain, T. Nishida, and L. Cattafesta, "Characterization of a Micromachined Thermal Shear Stress Sensor", AIAA J., 40, 6 (2002), pp. 1099-1104.

14. M. Sheplak, A. Padmanabhan, M. A. Schmidt, and K. S. Breuer, "Dynamic Calibration of a Shear Stress Sensor Using Stokes Layer Excitation", AIAA J., 39, 5 (2001), pp. 819-23.

15. D. Hyman, T. Pan, E. Reshotko, and M. Mehregany, "Microfabricated Shear Stress Sensors, Part 2: Testing and Calibration", AIAA J., 37, 1 (1999), pp. 73-8. 